\title{
1 Reliability assessment of null allele detection: inconsistencies between and within \\ 2 different methods
}

3

4 M.J. Dąbrowski ${ }^{1,2}$, M. Pilot ${ }^{1,3}$, M. Kruczyk ${ }^{2,4}$, M. Żmihorski ${ }^{1}$, H.M. Umer ${ }^{2}$, J. Gliwicz ${ }^{1}$

$5{ }^{1}$ Museum and Institute of Zoology, Polish Academy of Sciences, Wilcza 64, 00-679 Warsaw,

6 Poland

$7{ }^{2}$ Department of Cell and Molecular Biology, Uppsala University, Box 596, 75124 Uppsala,

8 Sweden

$9{ }^{3}$ School of Life Sciences, University of Lincoln, Brayford Pool, Lincoln LN6 7TS, UK

$10{ }^{4}$ Postgraduate School of Molecular Medicine, Zwirki i Wigury 61, 02-091 Warsaw, Poland

11 Keywords: genotyping errors, heterozygosity, Microtus oeconomus, microsatellite loci, null

12 alleles; bottleneck

13 Corresponding author: M.J. Dąbrowski, Museum and Institute of Zoology, Polish Academy of

14 Sciences, Wilcza 64, 00-679 Warsaw, Poland; Fax:+48 22 6296302; michal@ miiz.waw.pl

20 Running title: Assessment of null allele estimation methods

21 


\section{Abstract}

Microsatellite loci are widely used in population genetic studies, but the presence of

25 null alleles may lead to biased results. Here we assessed five methods that indirectly detect

26 null alleles, and found large inconsistencies among them. Our analysis was based on 20

27 microsatellite loci genotyped in a natural population of Microtus oeconomus sampled during 8

28 years, together with 1200 simulated populations without null alleles, but experiencing

29 bottlenecks of varying duration and intensity, and 120 simulated populations with known null

30 alleles. In the natural population, $29 \%$ of positive results were consistent between the methods

31 in pairwise comparisons, and in the simulated dataset this proportion was $14 \%$. The positive

32 results were also inconsistent between different years in the natural population. In the null-

33 allele-free simulated dataset, the number of false positives increased with increased bottleneck

34 intensity and duration. We also found a low concordance in null allele detection between the

35 original simulated populations and their $20 \%$ random subsets. In the populations simulated to

36 include null alleles, between $22 \%$ and $42 \%$ of true null alleles remained undetected, which

37 highlighted that detection errors are not restricted to false positives. None of the evaluated

38 methods clearly outperformed the others when both false positive and false negative rates

39 were considered. Accepting only the positive results consistent between at least two methods

40 should considerably reduce the false positive rate, but this approach may increase the false

41 negative rate. Our study demonstrates the need for novel null allele detection methods that

42 could be reliably applied to natural populations. 


\section{Introduction}

Highly polymorphic microsatellite markers are widely applied in population genetic

47 studies since their discovery in the late 1980s. The improvement of polymerase chain reaction 48 (PCR) and sequencing technologies allowed the use of these molecular markers to spread fast 49 and wide into many research fields (see Guichoux et al. 2011 for review). However, the 50 potential occurrence of "null alleles", i.e. alleles that fail to amplify during the PCR, creates a 51 disadvantage in using these markers (Oddou-Muratorio et al. 2009). A null allele occurs when 52 an incompatibility between any of the two locus-specific primers and its complementary 53 target region causes the PCR amplification of an allele to fail. Such incompatibilities may be 54 caused by mutations in the primer target region within one species, or between different 55 species (in case of cross-species amplification) (Callen et al. 1993, Primmer et al. 1995, Jarne 56 \& Lagoda 1996). In some cases, long alleles may amplify much less efficiently then shorter 57 ones, and therefore may appear as null alleles (Wattier et al. 1998). Low template 58 quality/quantity can also result in the absence of amplification product and may be interpreted 59 as the presence of a null allele (Garcia de Leon et al. 1998).

60 Null alleles have been reported in many species, e.g. humans (Callen et al. 1993), 61 deers (Pemberton et al. 1995), bears (Paetkau \& Strobeck 1995), voles (Ishibashi et al. 1996), 62 fish (McCoy et al. 2001), crayfish (Walker et al. 2002), and oystercatchers (Van Treuren 63 1998). The detection of null alleles is an important step in population genetic data analysis, as 64 their presence may strongly bias the estimates of population genetics parameters (Pemberton 65 et al. 1995, Chapuis \& Estoup 2007). For example, the accuracy of assignment of individuals 66 to populations may be reduced and $\mathrm{F}_{\mathrm{ST}}$ significantly overestimated (Carlsson 2008). The 
67 presence of null alleles may also lead to an incorrect exclusion of a significant number of true 68 parents in parentage analyses (Dakin \& Avise 2004). Despite this, very few studies on 69 population genetic structure and genetic parentage report estimates of null allele frequencies 70 in their data (see Dakin \& Avise 2004 for review).

71 Several methods for null allele estimation are currently available (Dempster et al. 72 1977, Chakraborty et al. 1992, Brookfield 1996, Summers \& Amos 1997, Kalinowski \& Taper 73 2006). They are based on comparing observed and expected heterozygosity for each locus to 74 identify loci with significant heterozygote deficit. This approach is based on the fact that a 75 heterozygous locus with a null allele would be scored as a homozygote, since only the visible 76 allele is detected. Crucially, all these methods assume that a population is in Hardy-Weinberg 77 Equilibrium (HWE), and that all observed deviations towards heterozygote deficit result from 78 the presence of null alleles. The main difference between these methods lies in the way blank 79 results (i.e. individuals without any detectable PCR product at a particular locus) are 80 interpreted. Some methods consider blank results as null allele homozygotes, while others 81 classify them as PCR failures resulting from low DNA quality or human errors; some methods 82 attempt to differentiate between these two cases (see Supplementary Material for details). 83 Another difference lies in the approaches used for null allele frequency estimation. While the 84 estimates of Chakraborty et al. (1992) and Brookfield (1996) are obtained analytically, 85 estimates of Dempster et al. (1977), Summers \& Amos (1997), and Kalinowski \& Taper 86 (2006) are achieved through iterative optimisation (see Supplementary Material for details).

87 The above methods showed good to moderate accuracy in estimating frequencies of 88 known null alleles in populations simulated assuming HWE (Kalinowski \& Taper 2006, 
89 Chapuis \& Estoup 2007). Specifically, Kalinowski \& Taper (2006) demonstrated that their 90 method performs better than the methods of Chakraborty et al. (1992) and Summers \& Amos 91 (1997), while Chapuis \& Estoup (2007) showed that the method of Dempster et al. (1977) 92 performs better than the methods of Chakraborty et al. (1992) and Brookfield (1996). 93 However, Chapuis \& Estoup (2007) also showed that the three methods they tested performed 94 worse when applied to two empirical datasets from natural populations, where the presence of 95 null alleles was confirmed by their successful amplification after the primers were re96 designed. Moreover, in one of these populations heterozygote deficit remained significant 97 even after the null allele was successfully amplified with the new primers. Although this result 98 was attributed to the presence of additional null alleles (Chapuis \& Estoup 2007), the 99 observed heterozygote deficit could have resulted from other factors such as small sample 100 size, high inbreeding levels, or immigration. 102 problematic when estimating null alleles in microsatellites scored from natural populations, 103 since natural populations never strictly comply with the assumptions of Hardy-Weinberg law 104 (i.e. infinite size, random mating, lack of mutations, migration and natural selection). 105 Crucially, some of the factors causing deviations from HWE also lead to heterozygote deficit, 106 namely inbreeding, assortative mating, population structure or immigration from a genetically 107 distinct source (Wahlund effect), and disruptive selection (Avise 2004). Heterozygote deficit 108 generated by such population mechanisms may be interpreted as the presence of null alleles, 109 thus leading to false positives. On the other hand, phenomena such as disassortative mating or 110 balancing selection can lead to heterozygote excess, which may result in failure to detect true 
111 null alleles. The effect of other population genetic processes is less obvious. For example, a 112 bottleneck leads to loss of alleles and decline in heterozygosity, but at least under some 113 conditions it may also lead to temporary heterozygote excess (Cornuet \& Luikart 1996). 114 Fluctuations in population size, especially if associated with immigration during the growth 115 phase, may lead to temporal fluctuations between heterozygote excess and deficit. In addition, 116 taking a small subsample from a population (which also effectively occurs during founder 117 events) may result in heterozygote deficit in some loci and heterozygote excess in others, due 118 to the stochasticity of the sampling procedure. This may lead to detection of false null alleles 119 in loci with heterozygote deficit.

120 Many population genetic studies are based on small sample sizes, and in many cases, 121 study populations themselves are small (and therefore subject to strong drift), fluctuate in size, 122 and exhibit considerable deviations from random mating. Such populations do not comply 123 with the assumption that heterozygote deficit results solely from the presence of null alleles. 124 However, the methods assuming HWE are commonly applied to such cases (e.g. see the 125 review by Dakin \& Avise 2004). In this study, we address the problem of detecting null alleles 126 in populations that undergo demographic changes and deviate from HWE, and we assess 127 reliability of the five widely used methods (Dempster et al. 1977, Chakraborty et al. 1992, 128 Brookfield 1996, Summers \& Amos 1997, Kalinowski \& Taper 2006) in such non-equilibrium 129 conditions. For this purpose, we apply these methods to a natural population of root vole, 130 Microtus oeconomus, which was sampled over an eight-year period, and underwent 131 substantial density fluctuations during this time. Additionally, in order to test whether 132 population-level factors may lead to the detection of false null alleles, we analysed 1200 
133 simulated populations without null alleles, but affected by a bottleneck with varying levels of 134 intensity and duration.

135

136 Materials and Methods

\section{Analysed datasets}

138 We analysed 20 nuclear microsatellite loci in a population of root vole, Microtus

139 oeconomus, which was extensively sampled over an eight-year period, and underwent a 7.7-

140 fold change in average density during this time. Detailed information about demography and

141 genetic variability of this population obtained from previous studies (Gliwicz \& Jancewicz

142 2004, Gliwicz \& Dąbrowski 2008, Dąbrowski 2010, Pilot et al. 2010) allowed us to follow

143 temporal changes in the estimated null allele frequencies and compare different methods of 144 their detection.

145 In order to assess the effect of demographic changes and resulting population genetic 146 changes on null allele detection rates under controlled conditions (i.e. with known - rather 147 than estimated - genetic composition and demographic history), we simulated 1200 148 populations without null alleles, but with varying level and duration of a bottleneck. The 149 simulated data allowed us to explore the effect of demographic changes on inconsistencies in 150 null allele detection that were observed in the natural population. In addition, we created $20 \%$ 151 subsets of the simulated populations by random sampling, to assess the effect of population 152 sub-sampling on null allele detection. Finally, we introduced null alleles into the earlier 153 simulated populations in order to (1) assess the performance of each method of null allele 154 estimation in detecting known null alleles, and (2) assess the empirical relationship between 
155 the frequency of null alleles and the frequency of null allele homozygotes in non-equilibrium 156 populations.

We used two general approaches for null allele detection. The first approach was based 158 on methods assessing heterozygote deficit, as described above (Dempster et al. 1977, 159 Chakraborty et al. 1992, Brookfield 1996, Summers \& Amos 1997, Kalinowski \& Taper 160 2006). We applied this approach to both the natural population and the simulated populations. 161 The second approach was based on the comparison of genotypes between parent-offspring 162 pairs, and was included here as the method that does not assume HWE. However, it could be 163 only applied to the natural population.

164

165 Sample collection from the natural population

The natural population studied was a wild population of the root vole inhabiting a river

167 valley located in a strict reserve of Białowieża National Park in north-eastern Poland. The 1168 ha field plot was situated on a vast open sedgeland, and was exposed to seasonal flooding. 169 The root vole is a small rodent with a maximum life span of 18 months (3 months on 170 average). In the studied population, individuals were reproductively active usually only for 171 one breeding season. We used a catch-mark-release (CMR) method, with at least three 172 trapping sessions carried out each year, using 100 live traps placed in a grid of 10 by $10 \mathrm{~m}$. 173 Mean trapping efficiency was over $90 \%$ of all individuals present on the plot (Pilot et al. 174 2010). The population underwent substantial density changes over the study period, with 175 average annual densities ranging from 9 to 69 individuals per hectare as estimated in MARK 176 software, and no individuals trapped in 2007 (Dąbrowski 2010). Such density fluctuations 
177 affected kin structure in the population and could be responsible for deviations from HWE 178 detected in some years (Pilot et al. 2010). 180 marked from 2000-2008, including 13 recaptures (originally marked in a previous year and 181 re-trapped in the next year; these samples were not duplicated in the genetic analyses). The 182 annual numbers of sampled individuals are presented in Table 1.

\section{Microsatellite genotyping in the wild root vole population}

185 Protocols for DNA extraction and microsatellite genotyping are described in detail in the 186 Supplementary Material. One crucial information to convey here is that there were no blank 187 results in this dataset, i.e. no individuals had missing data at any locus. Tissue samples were 188 obtained as biopsies and immediately stored in ethanol, which allowed us to work only with 189 DNA of good quality. PCR amplification was done using high-quality Taq polymerase 190 (included in QIAGEN Multiplex PCR Kit), and PCR reactions were repeated up to four times 191 for samples that initially failed (see Supplementary Material for details). This allowed us to 192 eliminate any missing data that could have resulted from low quality DNA, human errors and 193 PCR reagent failures. None of these steps would, however, eliminate missing data resulting 194 from the presence of null allele homozygotes. Given that our dataset did not contain any 195 missing data, we can state with a high confidence that no null allele homozygotes existed in 196 our dataset, which implies that null alleles, if present in this dataset, would only occur in low 197 frequencies. 
201 alleles per locus (N), observed (HO) and expected (HE) heterozygosity, mean polymorphic 202 information content (PIC) and exclusion probability for the first parent $(\operatorname{ExP}(1))$ were 203 calculated in CERVUS 3.0 (Marshall et al. 1998), while departures from HWE were estimated 204 for each locus in GENEPOP v 4.0.10 (Rousset 2008) (Supplementary Table 1). 206 methods: (1) the maximum likelihood (ML) estimator based on observed and expected 207 heterozygosities described by Chakraborty at al. (1992) with the modification of Brookfield 208 (1996) which accounts for the presence of null allele homozygotes, as implemented in 209 MICRO-CHECKER 2.2.1 (van Oosterhout et al. 2004); (2) the ML estimator using chi-square 210 goodness-of-fit, accounting for the presence of null allele homozygotes during optimization 211 rounds (Summers \& Amos 1997), as implemented in CERvUs 3.0; (3) the ML estimator 212 accounting for genotyping errors implemented in ML-NullFreq (Kalinowski \& Taper 2006); 213 (4) the ML method using iterative EM (expectation and maximization) of Dempster at al. 214 (1977) implemented in GENEPOP v4.0.10; (5) a method based on the comparison of 215 genotypes of parent-offspring pairs. The algorithms applied in each method (Dempster et al. 216 1977, Chakraborty et al. 1992, Brookfield 1996, Summers \& Amos 1997, Kalinowski \& Taper 217 2006) are described in the Supplementary Material. Hereafter the five methods will be 218 referred to by the names of the software packages that implement them, namely MICRO219 CHECKER, CERvus, ML-NullFreq, GENEPoP, and parent-offspring method. As a result of 220 testing our datasets using these methods, we obtained a binary response variable (presence- 
221 absence of null alleles) for each of the 20 loci and each of the five methods tested. In addition, 222 we tested for the presence of null alleles for the entire dataset with all years pooled (genotypes 223 of recaptured individuals were not duplicated; see Supplementary Material and 224 Supplementary Table 2).

225 The application of the parent-offspring method to the root vole population was 226 possible since a careful reconstruction of its kin structure was available from earlier studies 227 (Dąbrowski 2010, Pilot et al. 2010, see Supplementary Material). The program CERVUS 228 allows for a small number of mismatches between parent and offspring genotypes, if the 229 probability of the estimated relationship is high based on the conformity of the remaining loci. 230 Therefore, we could use mismatching loci to detect putative null alleles. We created a list of 231 parent-offspring pairs based on the results of the previous studies on this population 232 (Dąbrowski 2010, Pilot et al. 2010). The average rate of mismatches between parental and 233 offspring genotypes estimated using error rate analysis implemented in CERVUS was 0.08 (SD $234=0.097)$. Presence of a null allele in a locus was reported only if the observed mismatch in a 235 parent-offspring pair fitted the pattern expected by the presence of a null allele. For example, 236 if a female with genotype $\mathrm{AB}$ at a particular locus mates with a male with genotype $\mathrm{CN}$ 237 (where $\mathrm{N}$ is a null allele), $50 \%$ of their offspring are expected to have genotypes with this null 238 allele (either AN or BN). Visible genotypes of the father (CC) and the offspring (AA, BB) will 239 be inconsistent with the father-offspring relationship, therefore creating a mismatch at this 240 locus. In contrast to the methods based on the heterozygote deficit, the parent-offspring 241 method does not require the assumption of HWE. 
243 closely related individuals, which reduced the sample size to 511 parent-offspring pairs. In 244 contrast, the other four methods were tested using all sampled individuals. Due to smaller 245 sample size, the parent-offspring method may detect fewer null alleles compared with the 246 other methods. However, if each method detects null alleles correctly, the null alleles detected 247 by the parent-offspring method should be confirmed by the other methods.

249 Generation and analysis of simulated datasets

250 Using SPAms (Parreira et al. 2009), we simulated 1200 populations, each comprised 251 of 100 individuals with 20 loci. The reason for generating this data was to test the way 252 bottlenecks affect the detection of putative null alleles. In order to do this, the one population 253 size change model with instantaneous size change was applied with the following options: 254 ancestral pop effective diploid size: 100,000; present pop diploid size (six variants): (1) 255 99,999, (2) 50,000, (3) 25,000, (4) 10,000, (5) 5,000, (6) 2,500; duration of event (four 256 variants): 3, 30, 300, 3000 generations; and mutation rate: 0.0001 . The first size change 257 variant (from 100,000 to 99,999 individuals) was used as a control, where bottleneck effect 258 was not present. Each combination of the population size change (from ancestral to present 259 number of individuals) and time of this event was simulated in 50 replicates. In all 1200 260 simulated populations the presence of putative null alleles was tested using four programs: 261 Cervus, GenePoP, MICRO-CHECKER and ML-NullFreq. Genetic diversity in the simulated 262 populations was estimated using the same methods as for the natural population of root voles 263 (see above). To test the effect of random sampling on null allele detection, we randomly selected 
$26510 \%(\mathrm{n}=120)$ of the simulated populations, and from each of them we randomly sampled $26620 \%$ of individuals. This simulated the effects of three different real life scenarios: (A) 267 estimation of null allele frequencies based on a subset of individuals sampled from a 268 population, (B) founder effect, or (C) sudden change in number of individuals within one 269 breeding season. We tested for the presence of null alleles in each original population $(n=100$ 270 individuals) and its random subset ( $\mathrm{n}=20$ individuals, i.e. a $20 \%$ subset) separately. Then we 271 considered only the loci with null alleles detected in at least one of the original populations or 272 their subsets. For these populations, we calculated the Kendall's coefficient of concordance as 273 a measure of similarity of null allele detection between the original and the subset 274 populations.

For the next analysis, we selected 120 out of 1200 populations simulated in SPAmp, in 276 which no null alleles (false positives) were detected by any method. This set included 277 populations that underwent all levels of the simulated bottleneck. Then, we simulated the 278 presence of two null alleles in each of these 120 populations using NullAlleleGenerator 279 (http://www.lcb.uu.se/papers/dabrowski/NullAlleleGenerator.zip). NullAlleleGenerator randomly 280 selected a locus (out of the 20 loci simulated) and changed one random allele into a null 281 allele. This procedure was repeated for two loci, thus simulating two null alleles per 282 population. Whenever genotyped in heterozygous form, the allele selected as the null allele 283 was replaced by the other allele from that locus. Whenever occurring in a homozygous state, 284 the simulated null allele was marked as a blank result (missing data). This way we obtained 285 populations with true known null alleles, for which we assessed the performance of CERVUS, 286 GENEPOP, MICRO-CHECKER and ML-NullFreq in detecting null alleles. 
288 per population for the second time, but unlike in the first case, this time the 120 populations 289 were selected randomly. In this analysis, we checked the relationship between the frequency 290 of simulated null alleles and the frequency of null allele homozygotes. In a population under 291 HWE, the expected frequency of a null allele homozygote is $p^{2}$, where $p$ is the frequency of 292 the respective null allele. However, here we simulated populations that underwent a 293 bottleneck, and thus many of them deviated from HWE. Therefore, we checked empirically 294 how the frequency of null allele homozygotes depended on the frequency of null alleles. This 295 was needed for the interpretation of the lack of null allele homozygotes in the natural 296 population we studied (which also deviated from HWE and underwent substantial 297 demographic fluctuations).

\section{Statistical analysis}

300 In order to investigate the presence/absence of null alleles in a particular locus in 301 subsequent years, we applied a generalized linear mixed model (GLMM) with binomial error 302 distribution, and logit link function. We used this approach because several parameters can 303 potentially affect null allele presence and these parameters need to be included within one 304 statistical design. Moreover, as the study covers different years and different loci, both year 305 effect and loci effect have to be included as random variables to avoid pseudoreplications. We 306 thus used the occurrence pattern of null alleles (present vs. absent) as a dependent variable, 307 while the method of null allele estimation (marked as 1-5) was used as a fixed categorical 308 factor. Difference between observed $\left(\mathrm{H}_{\mathrm{O}}\right)$ and expected $\left(\mathrm{H}_{\mathrm{E}}\right)$ heterozygosity (hereafter $\left.\mathrm{H}_{\mathrm{O}} \mathrm{H}_{\mathrm{E}}\right)$, 
309 number of individuals trapped in a given year and number of alleles at a particular locus in a 310 given year were used as three separate covariates in the model, whereas year and locus were 311 included as random categorical factors. We implemented the GLMM using the "lmer" 312 package (Bates et al. 2011) in R (R Development Core Team 2011).

313 The expected number of loci with null alleles was estimated as a function of the 314 number of years analysed. For this purpose we used rarefaction curves implemented in 315 EstimateS 800 (Colwell 2005). The curves were based on the years resampled in a random 316 order. We constructed the curves independently for each method of null allele estimation. In order to assess how similar the five methods were in their estimates of null alleles 318 for a given locus in a particular year, we calculated a similarity index as the probability that a 319 null allele detected by one method will be confirmed by another. This index was calculated 320 pairwise between the methods, and visualized with $2 \times 2$ contingency tables.

322 independent of year and method $(8$ years $* 5$ methods $=40$ estimations; year 2007 was 323 removed due to lack of voles) using EstimateS 800 (Colwell 2005). In order to understand 324 what drives similarity among randomly selected null allele estimations, we carried out the 325 GLM analysis as follows: For each possible pair of estimations $(n=780$ pairs $)$ we computed: 326 (1) temporal distance (ranged from 0 to 8 years), (2) pooled number of null alleles indicated 327 by two estimations and (3) logical statement (yes or no) indicating whether both estimations 328 for a given pair were obtained with the same method (e.g. CERVUS vs. CERVUS) or different 329 method (CERVUS vs. GENEPOP). These three variables were used as explanatory variables, 330 whereas the number of shared null alleles in a given pair (indicating similarity level between 
331 two estimations compared, which ranged from 0 to 6 , mean $=0.77, \mathrm{SD}=0.95$ ) was used as 332 response variable in GLM with Poisson error distribution and log link in $\mathrm{R}$ package ( $\mathrm{R}$ 333 Development Core Team 2011). To assess whether a bottleneck influences the number of null alleles detected by each 335 method tested, we compared the number of detected null alleles between the no-bottleneck 336 variant with the five bottleneck variants using a $\chi^{2}$ test with Bonferroni correction. In order to 337 test whether the duration of the bottleneck affects the number of null alleles detected, we used 338 a Kruskal-Wallis test. To assess the level of consistency in null allele detection between the 339 tests applied to the entire population and to the corresponding randomly selected $20 \%$ subset, 340 we used Kendall's coefficient of concordance Wt implemented in R (R Development Core 341 Team 2011). All these calculations were carried out for each method of null allele detection.

343 assessed whether the observed frequency of null allele homozygotes in populations with 344 simulated null alleles (see Generation and analysis of simulated datasets), was significantly 345 different from the frequency expected under HWE.

\section{Results}

\section{Null allele detection in the root vole population}

The number of alleles per locus in the root vole population ranged from 4 to 25 (mean $350=14 ; \mathrm{SD}=5.6)$. Expected heterozygosity $\left(\mathrm{H}_{\mathrm{E}}\right)$ ranged from 0.107 to 0.925 (mean $=0.780 ; \mathrm{SD}$ $351=0.218)$, and observed heterozygosity $\left(\mathrm{H}_{\mathrm{O}}\right)$ from 0.080 to $0.926($ mean $=0.762 ; \mathrm{SD}=0.215)$. 352 The analysed loci had high mean polymorphic information content $($ mean $=0.760, \mathrm{SD}=$ 
3530.220 ) and high exclusion probability for the first parent (mean $=0.50, \mathrm{SD}=0.207$ ), which 354 allowed us to use them successfully in parentage and kinship analyses (see Pilot et al. 2010). 355 In all but one locus, significant deviations from HWE were detected in different years, with 356 both heterozygosity deficit (59\%) and excess (41\%) being observed (Supplementary Table 1). 357 In $60 \%$ of the loci $(n=12)$ the allele distribution had no missing alleles of any length within 358 the expected range. In $15 \%$ of the loci $(n=3)$, the allele distribution had one missing allele 359 length, other $15 \%(\mathrm{n}=3)$ had two missing allele lengths, and in the remaining $10 \%$ of the loci $360(\mathrm{n}=2)$, more than two allele lengths were missing. At the same time, we did not detect any 361 null allele homozygotes, which would be indicated as a locus with no detectable product 362 (blank result).

The number of loci in which putative null alleles were detected varied among years 364 and depended on the estimation method (Table 1). The set of 20 loci was analysed for each 365 study year separately, which resulted in 8 replicates and a total of 160 loci*replicates (number 366 of loci multiplied by number of years). In total, CERVUS detected the lowest number of 367 putative null alleles at 14 loci*replicates, while MICRO-CHECKER detected putative null 368 alleles in 15 loci*replicates, ML-NullFreq in 36 loci*replicates and GenePop in 46 369 loci*replicates. Altogether, using these four different methods we recorded 67 loci*replicates 370 with putative null alleles out of the total number of 160 loci*replicates. Among them, $68.5 \%$ $371(n=46)$ were detected only by one out of 4 methods, $12 \%(n=8)$ by 2 methods, $4.5 \%(n=3)$ 372 by 3 methods, and $15 \%(n=10)$ by 4 methods (Table 1$)$. Only two loci had no null alleles 373 detected in any year. There were no loci where null alleles were detected in each year (Table 374 1). Moreover, the number of loci with detected putative null alleles estimated for the entire 
375 dataset with all years pooled also depended on the estimation method (see Supplementary 376 Table 2). The frequency of detected putative null alleles (Table 1) was lower than $10 \%$ in $73 \%$ 377 of cases (Figure 1). We found no correlation between the number of discontinuities in allele 378 distribution and the number of detected null alleles within a locus. Finally, the frequency of 379 null allele detection at loci originally developed for $M$. oeconomus did not differ from the 380 frequency at loci originally developed for other species.

381

382 Null allele detection based on parent-offspring genotype comparison in the root vole 383 population

384 We investigated 270 father-offspring pairs and 241 mother-offspring pairs, resulting in 385 the detection of 18 parent-offspring pairs carrying putative null alleles. As shown in Table 1, 386 the parent-offspring method detected null alleles in 11 loci*replicates. Seven loci were 387 indicated as having null alleles once (in one out of 8 years), and 2 loci were indicated twice 388 (in two out of 8 years). Five loci*replicates with putative null alleles detected using the 389 parent-offspring analysis were also detected by all four ML programs tested (Table 1). Three 390 other loci*replicates indicated by the parent-offspring analysis were not confirmed by any of 391 these four programs. At the same time, four other loci*replicates with null alleles detected by 392 all the other four programs, were not confirmed by the parent-offspring analysis.

\section{Null allele detection in the simulated populations}

In the 1200 simulated populations the number of alleles per locus ranged from 2 to 34 $396($ mean $=14 ; \mathrm{SD}=4.2)$. Expected heterozygosity $\left(\mathrm{H}_{\mathrm{E}}\right)$ ranged from 0.068 to $0.957($ mean $=$ 
$3970.861 ; \mathrm{SD}=0.081)$, and observed heterozygosity $\left(\mathrm{H}_{\mathrm{O}}\right)$ from 0.07 to $1.0($ mean $=0.861 ; \mathrm{SD}=$ 3980.087 ). The analysed loci had high mean polymorphic information content (mean $=0.860 ; \mathrm{SD}$ $399=0.091)$ and high exclusion probability for the first parent $($ mean $=0.420, \mathrm{SD}=0.129)$ (for 400 detailed information see Supplementary Table 3). In some loci, significant deviations from 401 HWE were detected (Supplementary Table 4).

402 Among 24,000 loci*replicates, the number of loci with putative null alleles detected 403 was highest for ML-NullFreq ( $\mathrm{n}=1255$ loci*replicates; $5.2 \%$ of the total number) and 404 GENEPOP ( $\mathrm{n}=1123$ loci*replicates; 4.7\%), followed by MICRO-CHECKER ( $\mathrm{n}=500$ 405 loci*replicates; $2.1 \%$ ), and it was lowest for CERVUS ( $\mathrm{n}=327$ loci*replicates; $1.4 \%$ ) 406 (Supplementary Table 5). Altogether, using the four different methods we detected 2532 407 loci*replicates (10.5\% out of 24,000 analysed) with putative null alleles. Among them, $81 \%$ $408(n=2056)$ were detected only by one of the 4 methods, $12 \%(n=296)$ by 2 methods, $6 \%(n=$ $409163)$ by 3 methods, and $1 \%(n=17)$ by all 4 methods (Supplementary Table 4$)$.

$410 \quad$ All the null alleles detected in these 1200 simulated populations were false positives, as 411 the program SPAms used for their generation does not simulate null alleles. Therefore, we 412 selected 120 populations where no null alleles were detected, introduced simulated null alleles 413 by using NullAllelesGenerator, and repeated the analysis with the same four methods. In this 414 case, MICRO-CHECKER, CERVUS and ML-NullFreq detected either none or very low 415 frequencies of false positives ( $0.1 \%$ of loci that were actually free of null alleles and 1.6-1.9\% 416 of all loci with null alleles detected). In contrast, GENEPOP detected a considerable number of 417 false positives: they were found in $9 \%$ of loci that were actually free of null alleles and 418 constituted $55 \%$ of null alleles detected by this method (Table 2). Each of the four programs 
419 produced a considerable number of false negatives (i.e. true null alleles that remained 420 undetected) in proportions that ranged between 22\% (in ML-NullFreq) and $42 \%$ (in CERVUS) 421 of all loci with known true null alleles.

\section{$423 \underline{\text { Statistical analysis }}$}

The GLMM revealed that the probability of null allele detection in the root vole 425 population depended on the method applied. It was lowest for the parent-offspring method (as 426 expected due to smaller sample size used for this analysis - see Materials and Methods) and 427 highest for the GENEPOP method (Table 3). The number of null alleles detected using the 428 parent-offspring method was about 16 times lower as compared to GENEPOP and nearly 10 429 times lower as compared to ML-NullFreq method. Within the remaining methods, the 430 differences were also significant: the frequency of null allele detection was higher in ML431 NullFreq than in Cervus $(P<0.0001)$, and higher in GenePoP than in Cervus $(P<0.0001)$ 432 but no differences were found between CERVUS and MICRO-CHECKER $(P=0.673)$.

433 The GLMM also revealed that differences between observed and expected 434 heterozygosity $\left(\mathrm{H}_{\mathrm{O}} \mathrm{H}_{\mathrm{E}}\right)$ in the root vole population had a significant influence on the detection 435 probability of putative null alleles (Table 3 ). The effects of number of individuals trapped in a 436 particular year, and the number of alleles in a given locus in a particular year were non437 significant (Table 3).

438 Although the expected cumulative number of loci where putative null alleles were 439 detected increased asymptotically with increasing sample size, the rate of increase differed 440 between the five methods applied (Figure 2). Depending on the method, null alleles occurred 
441 in $25 \%$ to $75 \%$ of loci for the all eight years cumulatively.

Inspection of the similarity patterns shows that a null allele detected in the root vole

443 population by a given method is usually a very weak predictor of it being detected by another 444 method. As a consequence, consistent estimates of null alleles by two methods were rare and 445 ranged from $12 \%$ to $58 \%$ of method-pairs (mean $=29.05 \%$, Figure 3 ). In the simulated 446 populations, the observed similarity pattern was even lower and ranged from $1.8 \%$ to $35.4 \%$ $447($ mean $=13.6 \%$, Figure 3$)$.

448 GLMs showed that the pooled number of null alleles estimated in the root vole 449 population by two randomly selected methods explained the number of null alleles shared by 450 these two methods (GLM, $\mathrm{B}=0.19, \mathrm{SE}=0.01, \mathrm{z}=18.06, P<0.001)$. Contrary to 451 expectations, the number of shared putative null alleles was similar in the "between-methods" 452 and the "within-method" pairs of estimates $(\mathrm{B}=0.14, \mathrm{SE}=0.10, \mathrm{z}=1.36, P=0.174)$. The 453 effect of temporal distance between samples from different years was insignificant for the 454 similarity among estimates $(\mathrm{B}=-0.04, \mathrm{SE}=0.02, \mathrm{z}=1.85, P=0.064)$.

455 In the simulated populations ( $\mathrm{n}=100$ individuals each), a change in the population size 456 (bottleneck effect) significantly affected the number of loci with detected null alleles (Table 457 4). GENEPOP detected significantly higher number of loci with null alleles in four out of five 458 comparisons, CERVUS in three, and the remaining two programs in one (Table 4). We also 459 found that the cumulative number of loci with detected null alleles increased with the 460 increased bottleneck duration (Figure 4). Bottleneck duration also significantly affected the 461 number of null alleles detected by GENEPOP (Kruskal-Wallis $\mathrm{Hc}=11.9 ; P<0.05$ ), although 462 no significant correlation was detected in other programs. 
464 concordance of null alleles detected (using each of the four methods) as compared with the 465 original population $(\mathrm{n}=100$ individuals) (mean Kendall's coefficient of concordance for four 466 programs $\mathrm{Wt}=0.11 ; \mathrm{SD}=0.06)($ Table 5$)$.

$467 \quad$ Finally, in populations with simulated null alleles, the observed frequency of null allele 468 homozygotes differed significantly from the expected frequency $(\mathrm{V}=10070 ; \mathrm{P}<0.001$; 469 Supplementary Figure 1). We observed deviations toward both null allele homozygote 470 deficiency and excess (Supplementary Figure 1). For null allele frequencies below 0.17 we 471 observed cases were no null allele homozygotes occurred, but there were no such cases for 472 null allele frequencies higher than 0.17 .

\section{Discussion}

476 these methods are based on assumptions that are commonly violated in natural populations.

477 Methods based on comparing observed and expected heterozygosity (Dempster et al. 1977, 478 Chakraborty et al. 1992, Brookfield 1996, Summers \& Amos 1997, Kalinowski \& Taper 479 2006) assume that null alleles can be detected based on observed deviations from HWE 480 towards heterozygote deficit. However, natural populations may deviate from HWE because 481 they do not meet the assumptions of the Hardy-Weinberg law, and/or because they are often 482 studied based on a small number of samples, which may lead to random deviations from the 483 equilibrium at different loci. The parentage method does not assume HWE, but may be prone 484 to other types of errors, e.g. human errors with microsatellite scoring. The error rate in null 
485 allele detection in natural populations is difficult to estimate, because the actual null allele 486 frequencies are usually unknown. Our study was based on a natural population that was 487 sampled for several consecutive years, and so the same null alleles were expected to occur 488 throughout the entire study period. This allowed us to test the reliability of several methods of 489 null allele estimation through the comparison of results between different years. Application 490 of the same methods to 1200 simulated populations that underwent bottlenecks of different 491 intensity and duration allowed us to further examine the effect of strong genetic drift on null 492 allele detection.

493

\section{Accuracy of null allele detection in the root vole population}

495 We found inconsistencies in null allele estimation both across years for each method 496 and among different methods within each year. We also failed to find any statistically 497 significant temporal repeatability in null allele detection at any locus. Each method detected a 498 considerable number of null alleles in the 1200 simulated datasets, and there were significant 499 differences in null allele estimates among the methods. However, the number of null alleles 500 detected was positively correlated with the bottleneck size in each of the methods tested. 501 Crucially, the simulated populations did not originally include any null alleles (the program 502 SPAms used for their generation does not simulate null alleles), so all the detected null alleles 503 were false positives.

We thus conclude that all putative null alleles detected in the root vole population are 505 likely to be false positives. Our conclusion is supported by the following evidence: First, 506 given that real null alleles are derived from primer compatibility problems during PCR 
507 amplification and PCR protocols did not change between years, we would expect the same 508 null alleles to be present in each year of the study, or at least in most years (accounting for the 509 sampling effect - see below). Yet, none of the methods tested detected such continuous 510 presence for any of the loci. One explanation for this could be that individuals having null 511 alleles in their genotypes were not sampled every year. In that case the number of individuals 512 sampled in a particular year should have a significant influence on null allele detection 513 probability. The GLMM analysis did not find such a correlation, which allows us to reject this 514 explanation. Additionally, according to MARK estimate, over 90\% of all individuals present 515 in the study population were genotyped, and therefore the probability of omitting all 516 individuals with a given null allele is negligible, unless this allele has a very low frequency in 517 the population. However, the impact of null alleles with such low frequencies on results of 518 population-level genetic analyses would be negligible.

520 low frequency or lack of null alleles in this population. Although the relationship between null 521 allele frequency and the frequency of null allele homozygotes based on the Hardy-Weinberg 522 law does not necessarily hold in non-equilibrium populations, these two parameters are 523 always dependent as demonstrated for the simulated populations. Therefore, it is expected that 524 in a locus with high null allele frequency, some blank results should occur. There are some 525 cases in the root vole population where the estimated null allele frequency was above $20 \%$ 526 (Figure 1). Under the Hardy-Weinberg law, such loci should contain over 4\% of null allele 527 homozygotes. However, we detected none, despite a large number of genotyped individuals. Third, kin clustering and non-random mating have been earlier demonstrated in this 
529 population (Dąbrowski 2010). We also found that significant multi-annual changes in density 530 and random environmental events (e.g. seasonal floods) have a strong impact on rates of 531 seasonal migration, male dispersal, and female philopatry (Pilot et al. 2010, Dąbrowski 2010). 532 Heterozygosity could thus have been lost (and regained) from year to year due to both genetic 533 drift and migration (Pilot et al. 2010, Dąbrowski 2010). We also found genetic signatures of 534 bottleneck in this population (Pilot et al. 2010), and we show in this study, based on simulated 535 data, that bottlenecks may significantly increase the frequency of false null alleles detected by 536 each of the methods tested. Therefore, we conclude that the pattern of the putative null allele

537 occurrence observed in the study population, is more likely to result from population genetic 538 processes like density fluctuations, migration and non-random mating, than from factors 539 associated with PCR amplification outcomes.

540

541 Inconsistencies among different methods of null allele detection

542 Our study revealed large inconsistencies among the compared methods of null allele 543 detection in both the natural root vole population and the simulated datasets. The average 544 similarity among the methods used to detect null alleles was $29.05 \%$ for the root vole 545 population and only $13.6 \%$ for the simulated populations. While detection of false null alleles 546 may be explained by population genetic processes leading to deviations from HWE, 547 inconsistencies among the methods cannot be accounted for solely by this explanation. The 548 method based on parentage analysis relied on different assumptions and smaller pool of 549 individuals than the heterozygosity-based methods, and the resulting differences were 550 consistent with expectations. However, the four heterozygosity-based methods applied the 
551 same general assumptions (see Supplementary Material). They differed in the way missing 552 data was interpreted, but neither the root vole population nor the original SPAms-generated 553 populations included any missing data. Therefore, we conclude that the discrepancies among 554 these methods do not result from differences in the theoretical assumptions, but rather from 555 differences in the particular optimisation algorithms applied.

556

557 How to combine different methods to minimise errors in null allele detection?

558 Our study raises a question regarding whether estimates of null alleles reported in the 559 literature, which are usually inferred using indirect methods, are always reliable. The number 560 of null allele occurrences within different allele frequency classes calculated in this study for 561 the root vole population (Figure 1) has a similar distribution to the one shown in Dakin \& 562 Avise (2004), based on an extensive literature review. Given that our results show that most 563 null alleles detected in the root vole population are likely false positives (see above), this 564 similarity raises a further question of whether the recommendation for discarding loci 565 showing null alleles from analysed datasets (De Sousa et al. 2005) should be followed 566 unconditionally. 568 have to be excluded following this recommendation, with different number of loci excluded 569 depending on the year and the detection method used. Moreover, if sampling was carried out 570 for a longer period, we may expect that the number of loci with putative null alleles would 571 increase with the number of study years (see Figure 2), because we observed no consistent 572 detection pattern among years for any locus. 

574 errors in null allele detection based on the results from our study. We found that in the 575 simulated populations without null alleles, $81 \%$ of false positives were detected by only one 576 out of the four heterozygosity-based methods, while only $1 \%$ of false positives were detected 577 by all the four methods. At the same time, in the simulated populations where null alleles 578 were included, $58 \%$ of true null alleles were detected by all the four methods. Therefore, 579 combining two or more methods and considering only the consistent putative null alleles 580 should considerably reduce the detection of false positives. However, it may also result in 581 non-detection of some true null alleles, especially if more than two methods are applied. Therefore, it may be useful to assess which of the four methods tested are less error583 prone. In the simulated populations without null alleles, CERVUS and MICRO-CHECKER 584 detected less false positives (1.4\% and $2.1 \%$, respectively) as compared with the two other 585 methods. On the other hand, in the simulated populations with null alleles, ML-NullFreq had 586 the lowest proportion of false negatives (22\%), while for CERVUS and MICRO-CHECKER 587 this proportion was $42 \%$ and $33 \%$, respectively. GENEPOP was the only method that still 588 detected a considerable number of false positives in the simulated datasets (prior to the 589 simulation of true null alleles) that were pre-selected specifically as having no false positives 590 detected by any of the four programs. Therefore, this program seems to be particularly error591 prone in terms of the detection of false null alleles. We thus suggest that the best strategy to 592 minimise the errors in null allele detection would be the combined use of two or three of the 593 remaining methods (ML-NullFreq, CERVUS and MICRO-CHECKER). The combination of 594 CERVUS and MICRO-CHECKER is best for minimising the false positives' rate, while the 
595 combination of ML-NullFreq and MICRO-CHECKER is best for minimising the false 596 negatives' rate.

598 of other types of genotyping errors like allelic dropouts or false alleles (e.g. resulting from 599 stuttering), which can be detected e.g. using MICRO-CHECKER and/or by replicating the 600 genotyping for a number of individuals. It is also important to minimise the occurrence of 601 missing data due to reasons other than null allele homozygotes by repeating failed PCRs at 602 least once. Because heterozygosity-based methods assume HWE, it is important to minimise 604 errors that may result from violations of the assumptions of Hardy-Weinberg law. For 605 example, if population genetic structure is detected, the presence of null alleles should be 606 assessed for each sub-population separately. The parentage-based method does not assume 607 HWE, so it may help minimising the detection of false null alleles if used in addition to the 608 heterozygosity-based methods; however, we recognise it won't always be possible or practical 609 to use this method, due to its reliance on a detailed reconstruction of parent-offspring 610 relationships within the study population. Finally, our study showed that material collected 611 from the same population during several seasons (if there is sufficient generational turnover) 612 may help interpreting the results of null allele detection and prevent their overestimation of 613 their numbers. Alternatively, if sample size is sufficiently large, the accuracy of null allele 614 detection may be improved by comparing the results obtained from different random sub-sets 615 of the entire dataset analysed. 


\section{Conclusions}

618 Our study shows that many commonly used null allele detection methods exhibit low

619 reliability and consistency when applied to non-equilibrium populations. When we account

620 for both false null allele detection rate and non-detection rate of the true null alleles, no

621 method can be considered as clearly superior over the others. We thus suggest the combined

622 use of at least two methods and considering only putative null alleles detected consistently by

623 different methods. This should considerably reduce the detection of false positives. However,

624 this approach is compromised by an increased rate of false negatives (non-detected real null

625 alleles), and thus provides only a sub-optimal solution. Our study demonstrates the need to

626 develop null allele detection methods that could be applied to non-equilibrium populations

627 without violating the model assumptions.

628

\section{Acknowledgements}

630 We are grateful to Dr M. Ratkiewicz for the inspiration for this study. We thank Prof. Oscar 631 Gaggiotti, Dr Andre Moura and three anonymous reviewers for their constructive comments 632 on the earlier version of this manuscript. We also would like to thank Dr E. Jancewicz and the 633 students from the Faculty of Forestry, Warsaw University of Life Sciences - SGGW for 634 assistance in the sample collection. The study was supported by a grant NN304 346339 635 (awarded to J. Gliwicz) from Polish National Science Centre. M. Pilot was supported by a 636 fellowship from the Foundation for Polish Science. 


\section{References}

638 Avise JC (2004) Molecular Markers, Natural History, and Evolution, 2nd edn. Sinauer 639 Associates Inc., Sunderland.

640 Bates D, Maechler M, Bolker B (2011) lme4: Linear mixed-effects models using S4 classes.R 641 package version 0.999375-42.http://CRAN.R-project.org/package=lme4

642 Brookfield JFY (1996) A simple new method for estimating null allele frequency from 643 heterozygote deficiency. Molecular Ecology, 5, 453-455.

644 Callen DF, Thompson AD, Shen Y, Phillips HA, Richards RI, Mulley JC, Sutherland GR 645 (1993) Incidence and origin of null alleles in the (AC)n microsatellite markers. American 646 Journal of Human Genetics, 52, 922-927.

647 Carlsson J (2008) Effect of microsatellite null alleles on assignment testing. Journal of 648 Heredity, 99, 616-623.

649 Chakraborty R, De Andrade M, Daiger SP, Budowle B (1992) Apparent heterozygote 650 deficiencies observed in DNA typing data and their implications in forensic applications. 651 Annals of Human Genetics, 56, 45-57.

652 Chapuis MP, Estoup A (2007) Microsatellite null alleles and estimation of population 653 differentation. Molecular Biology and Evolution, 24, 621-631.

654 Colwell RK (2005) EstimateS: Statistical estimation of species richness and shared species 655 from samples. Version 7.5. User's Guide and application published at: 656 http://purl.oclc.org/estimates.

657 Cornuet JM, Luikart G (1996) Description and power analysis of two tests for detecting recent 658 population bottlenecks from allele frequency data. Genetics,144,2001-2014. 
659 Dąbrowski MJ (2010) Relatedness, space use strategies and density changes in the population 660 of the root vole Microtus oeconomus. PhD thesis, Museum and Institute of Zoology PAS,

661 Warsaw.

662 Dakin EE, Avise JC (2004) Microsatellite null alleles in parentage analysis. Heredity, 93, 504663509.

664 De Sousa SN, Finkeldey R, Gailing O (2005) Experimental verification of microsatellite null 665 alleles in Norway spruce (Picea abies [L.] Karst.): Implications for population genetics 666 studies. Plant Molecular Biology Reporter, 23, 113-119.

667 Dempster AP, Laird NM, Rubin DB (1977) Maximum likelihood from incomplete data via the 668 EM algorithm. Journal of the Royal Statistical Society. Series B (Methodological), 39, 1-38.

669 Garcia de Leon FJ, Canonne M, Quillet E, Bonhomme F, Chatain B (1998) The application of 670 microsatellite markers to breeding programmes in the sea bass, Dicentrarchus labrax. 671 Aquaculture, 159, 303-316.

672 Gliwicz J, Dąbrowski MJ (2008) Ecological factors affecting the dial activity of voles in a 673 multi-species community. Annales Zoologici Fennici, 45, 242-247.

674 Gliwicz J, Jancewicz E (2004) Voles in river valleys. In: Jędrzejewska B and Wójcik JM 675 (eds.) Essays on Mammals of Białowieża Forest, 139-148. MRI PAS, Białowieża.

676 Guichoux E, Lagache L, Wagner S et al. (2011) Current trends in microsatellite genotyping. 677 Molecular Ecology Resources, 11, 591-611.

678 Ishibashi Y, Saitoh T, Abe S, Yoshida MC (1996) Null microsatellite alleles due to nucleotide 679 sequence variation in the grey-sided vole Clethrionomys rufocanus. Molecular Ecology, 5, $680589-590$. 
681 Jarne P, Lagoda PJL (1996) Microsatellites, from molecules to populations and back. Trends 682 in Ecology \& Evolution, 11, 424-429.

683 Kalinowski ST, Taper ML (2006) Maximum likelihood estimation of the frequency of null 684 alleles at microsatellite loci. Conservation Genetics, 7, 991-995.

685 Marshall TC, Slate J, Kruuk LEB, Pemberton JM (1998) Statistical confidence for likelihood686 based paternity inference in natural populations. Molecular Ecology, 7, 639-655.

687 McCoy EE, Jones AG, Avise JC (2001) The genetic mating system and tests for cuckoldry in a 688 pipefish species in which males fertilize eggs and brood offspring externally. Molecular 689 Ecology, 10, 1793-1800.

690 Oddou-Muratorio S, Vendramin GG, Buiteveld J, Fady B (2009) Population estimator of 691 progent test: what is the best method to assess null allele frequencies at SSR loci? 692 Conservation Genetics, 10, 1343-1347.

693 van Oosterhout C, Hutchinson WF, Wills DMP, Shipley P (2004) MICRO-CHECKER: 694 software for identifying and correcting genotyping errors in microsatellite data. Molecular 695 Ecology Notes, 4, 535-538.

696 Paetkau D, Strobeck C (1995) The molecular basis and evolutionary history of a microsatellite 697 null allele in bears.Molecular Ecology, 4, 519-520.

698 Parreira B, Trussart M, Sousa V, Hudson R and Chikhi L (2009) SPAms: A user-friendly 699 software to simulate population genetics data under complex demographic models. Molecular 700 Ecology Resources, 9, 749-753.

701 Pemberton JMS, Slate J, Bancroft DR, Barrett JA (1995) Non-amplifying alleles at 702 microsatellite loci: a caution for parentage and population studies. Molecular Ecology, 4, 249- 
703252.

704 Pilot M, Dąbrowski MJ, Jancewicz E, Schtickzelle N and Gliwicz J (2010) Temporally stable 705 genetic variability and dynamic kinship structure in a fluctuating population of the root vole 706 Microtus oeconomus. Molecular Ecology, 19, 2800-2812.

707 Primmer CR, Moller AP, Ellegren H (1995) Resolving genetic relationship with microsatellite 708 markers: a parentage testing system for the swallow Hirundo rustica. Molecular Ecology, 4, $709493-498$.

710 R Development Core Team (2011) R: A language and environment for statistical computing. R 711 Foundation for Statistical Computing, Vienna, Austria. ISBN 3-900051-07-0, URL 712 http://www.R-project.org/.

713 Rousset F (2008) Genepop'007: a complete reimplementation of the Genepop software for 714 Windows and Linux. Molecular Ecology Resources, 8, 103-106.

715 Summers K, Amos W (1997) Behavioral, ecological, and molecular genetic analyses of 716 reproductive strategies in the Amazonian dart-poison frog, Dendrobates ventrimaculatus. 717 Behavioral Ecology, 8, 260-267.

718 Van Treuren R (1998) Estimating null allele frequencies at a microsatellite locus in the 719 oystercatcher (Haematopus ostralegus). Molecular Ecology, 7, 1413-1417.

720 Walker D, Porter BA, Avise JC (2002) Genetic parentage assessment in the crayfish 721 Orconecte splacidus, a high-fecundity invertebrate with extended maternal brood care.

722 Molecular Ecology, 11, 2115-2122.

723 Wattier R, Engel CR, Saumitou-Laprade P, Valero M (1998) Short allele dominance as a 724 source of heterozygote deficiency at microsatellite loci: experimental evidence at the 
725 dinucleotide locus Gv1CT in Gracilaria gracilis (Rhodophyta). Molecular Ecology, 7, 1569-

7261573.

727

728

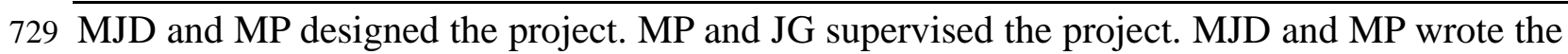
730 paper. MJD performed laboratory work, generated simulated populations and carried out null 731 allele detection analysis. MK designed and implemented the algorithm for simulating null 732 alleles, contributed to the statistical analyses and editing of the manuscript. MZ designed and 733 conducted most of the statistical analyses and contributed to writing the manuscript. HMU 734 participated in programming and automating of the process of comparing null allele detection 735 results. This project used data collected as a result of a long-term research project on rodents 736 in Białowieża National Park supervised by JG.

\section{Data Accessibility}

740 Data underlying this manuscript are available in the online supplemental information.

741 Microtus oeconomus genotypes, simulated populations genotypes and subpopulations 742 genotypes are available on Dryad, doi: 10.5061/dryad.4p41m

743 NullAlleleGenerator, along with its full documentation and example data is available from 744 http://www.lcb.uu.se/papers/dabrowski/NullAlleleGenerator.zip 


\section{Figure legends}

747 Figure 1. Histogram of frequencies of putative null alleles detected using different algorithms

748 implemented in the evaluated programs: CERVUS (algorithm of Summers \& Amos); MICRO-

749 CHECKER (algorithms: Oosterhout, Chakraborty, Brookfield 1, Brookfield 2), and GENEPoP

750 (EM algorithm of Dempster 1977).

751

752 Figure 2. Expected cumulative number (left axis) and percentage (right axis) of loci where

753 putative null alleles were detected using five different methods, are presented as a function of 754 the number of years studied. C - CERVus; M - MICRO-CHECKER; N - ML-NullFreq; G 755 GENEPOP.

756

757 Figure 3. Similarity of null allele estimates between the methods applied for the natural root 758 vole population and the simulated populations. The plot gives an average expectation that a 759 null allele detected by one method will also be detected by the other method (gray - the 760 simulated data; white - the root vole data).

761

762 Figure 4. Cumulative number of loci with putative null alleles within the simulated 763 populations $(\mathrm{n}=100$ individuals each) with different bottleneck scenarios. "Time" denotes 764 the bottleneck duration in generations. Cumulative number of loci is the sum of loci where 765 null alleles were detected using any of the four methods. 
767 Table 1. The presence of putative null alleles in the root vole population in each locus per 768 year, estimated using five different methods.

\begin{tabular}{|c|c|c|c|c|c|c|c|c|c|}
\hline Study year & 2000 & 2001 & 2002 & 2003 & 2004 & 2005 & 2006 & 2007 & 2008 \\
\hline Sample size & 150 & 116 & 130 & 39 & 70 & 147 & 84 & 0 & 16 \\
\hline \multicolumn{10}{|l|}{ Locus } \\
\hline AV12 & & & $\mathrm{N}$ & $\mathrm{N}$ & G & $\mathrm{P}$ & & & $\mathrm{G}$ \\
\hline AV13 & CMNG & & CMNGP & CMNG & NG & & & & \\
\hline AV14 & & & & NG & & $\mathrm{N}$ & $\mathrm{G}$ & & \\
\hline AV15 & $\mathrm{N}$ & & & & & & & & \\
\hline Moe1 & & $\mathrm{G}$ & M & $\mathrm{G}$ & & $\mathrm{G}$ & $\mathrm{N}$ & & $\mathrm{G}$ \\
\hline Moe2 & & & & $\mathrm{G}$ & & $\mathrm{G}$ & & & \\
\hline Moe3 & G & NG & $\mathrm{G}$ & & & MNG & $\mathrm{G}$ & & \\
\hline Moe4 & CMNGP & NG & & CMN & $\mathrm{N}$ & & CMNGP & & $\mathrm{N}$ \\
\hline Moe5 & & $\mathrm{P}$ & & & $\mathrm{N}$ & & & & \\
\hline Moe6 & $\mathrm{MN}$ & G & $\mathrm{N}$ & G & $\mathrm{P}$ & & $\mathrm{N}$ & & $\mathrm{CNG}$ \\
\hline Moe7 & & CMNGP & MG & $\mathrm{G}$ & & CMNG & CMNGP & & $\mathrm{G}$ \\
\hline MSCRB4 & & & $\mathrm{C}$ & $\mathrm{C}$ & & & & & \\
\hline MSCRB6 & & G & & $\mathrm{G}$ & & & & & \\
\hline \multicolumn{10}{|l|}{ MSMM2 } \\
\hline MSMM3 & $\mathrm{N}$ & & $\mathrm{P}$ & & $\mathrm{N}$ & $\mathrm{N}$ & & & \\
\hline MSMM4 & $\mathrm{N}$ & & & $\mathrm{N}$ & & NG & G & & \\
\hline MSMM5 & NG & $\mathrm{G}$ & G & G & G & $\mathrm{G}$ & & & \\
\hline MSMM6 & & CMNGP & & G & & CMNG & & & $\mathrm{G}$ \\
\hline MSMM7 & NP & & & $\mathrm{G}$ & & & & & \\
\hline MSMM8 & & & & & & & & & \\
\hline
\end{tabular}

769 The presence of a putative null allele is marked by the symbol of the program (or multiple programs) that

770 detected it: C - CERVUS; M - MICRO-CHECKER; N - ML-NullFreq; G - GENEPOP, and P - comparison of 771 mismatching loci in parent-offspring genotypes. In the second row, the number of individuals sampled in each 772 year is shown (Sample size). 
774 Table 2. Null alleles detected using MICRO-CHECKER, CERvUS, ML-NullFreq and 775 GENEPOP for 120 simulated populations containing two null alleles each. Loci with known 776 null alleles were compared with loci detected using different programs (0- loci without null 777 alleles; 1- loci with null alleles). Black background: true positives, grey background: true 778 negatives, white background: false negatives, underline value: false positives.

779

\begin{tabular}{lccc} 
& \multicolumn{3}{c}{ Null Alleles Generator } \\
& & 0 & 1 \\
\hline MICRO-CHECKER & 0 & 2164 & 78 \\
& 1 & $\underline{2}$ & 156 \\
\hline CERVUS & 0 & 2166 & 99 \\
& 1 & $\underline{0}$ & 135 \\
\hline ML-NullFreq & & & \\
& 0 & 2163 & 52 \\
& 1 & $\underline{3}$ & 182 \\
\hline GENEPOP & & & 162
\end{tabular}


780 Table 3. Summary results of a generalized linear mixed model with binomial error 781 distribution and logit link, explaining the presence of the null alleles as a function of the four 782 predictors: (1) difference between observed and expected heterozygosity $\left(\mathrm{H}_{\mathrm{O}} \mathrm{H}_{\mathrm{E}}\right),(2)$ number 783 of individuals trapped in a given year, (3) number of alleles in a particular locus in a given 784 year and (4-8) the method of null allele detection. Symbols of different methods are explained 785 in Table 1. Year and locus were included as random categorical factors in the model. For 786 every level of each predictor the following parameters are given: estimate (B), with standard 787 errors (SE), exponentiated estimate $(\operatorname{Exp}(B))$, tests statistic (z-value), and significance $(P$ 788 value).

\begin{tabular}{lrrrrr}
\hline Effect & $\mathrm{B}$ & $\mathrm{SE}$ & $\operatorname{Exp}(\mathrm{B})$ & $\mathrm{z}$-value & $P$-value \\
\hline Intercept & -3.507 & 0.593 & 0.030 & -5.918 & 0.000 \\
& -28.760 & 3.070 & 0.000 & -9.368 & $<0.001$ \\
(1) $\mathrm{H}_{\mathrm{O}} \mathrm{H}_{\mathrm{E}}$ & 0.000 & 0.003 & 1.000 & -0.013 & 0.990 \\
(2) N of individuals & -0.037 & 0.031 & 0.964 & -1.175 & 0.240 \\
(3) N of alleles & 0.579 & 0.554 & 1.785 & 1.045 & 0.296 \\
(4) Method = G & 2.782 & 0.495 & 16.154 & 5.623 & $<0.001$ \\
(5) Method = M & 0.811 & 0.541 & 2.249 & 1.498 & 0.134 \\
(6) Method = N & 2.283 & 0.498 & 9.806 & 4.586 & $<0.001$ \\
(7) Method = P & & & 1.000 & & \\
(8) Method = C & 0.000 & & & & \\
\hline
\end{tabular}


789 Table 4. Pairwise comparisons of the number of loci with putative null alleles between 790 variant 1 of the simulation (no bottleneck) with the other five variants with different level of 791 bottleneck. Comparisons were made for each program separately. Values presented in the 792 table are results of $\chi^{2}$ test. Statistically significant results are marked with (*).

\begin{tabular}{lllll}
\hline Bottleneck pair variants & CERVUS & GENEPOP & MICRO-CHECKER & ML-NullFreq \\
\hline $1-2$ & 0.272 & 2.010 & 0.286 & $<0.001$ \\
$1-3$ & 2.502 & $70.040^{* *}$ & 3.704 & 4.560 \\
$1-4$ & $9.318^{*}$ & $148.700^{* *}$ & 0.008 & 0.011 \\
$1-5$ & $42.760^{* *}$ & $206.500^{* *}$ & 1.347 & 1.612 \\
$1-6$ & $114.700^{* *}$ & $176.500^{* *}$ & $24.940^{* *}$ & $16.870^{*}$ \\
\hline
\end{tabular}

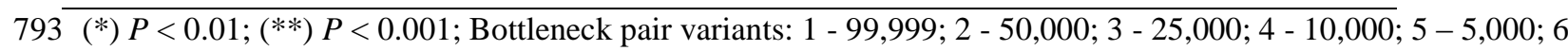
$794-2,500$. The ancestral number of individuals for all bottleneck variants was set to 100,000 individuals. 
795 Table 5. Pairwise comparison of loci with detected null alleles in two data sets: simulated

796 original populations ( $\mathrm{n}=100$ individuals per each population) and sub-sampled populations

797 ( $\mathrm{n}=20$ individuals randomly selected from original population).

\begin{tabular}{lllll}
\hline Variable & CERVUS & GENEPOP & MICRO-CHECKER & ML-NullFreq \\
\hline $\mathrm{Wt}$ & 0.182 & 0.040 & 0.093 & 0.123 \\
$\mathrm{~N}$ & 229 & 223 & 59 & 214 \\
$\mathrm{CP}$ & 13 & 9 & 6 & 30 \\
new-P & 204 & 129 & 24 & 96 \\
not-CP & 12 & 85 & 29 & 88 \\
\hline
\end{tabular}

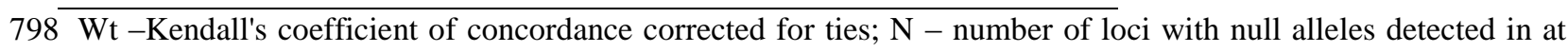
799 least one dataset (either original or under-sampled); CP - conserved positives: loci with detected null alleles in 800 both sets of populations; new-P - new positives: loci with null alleles detected only in under-sampled data set; 801 not-CP - not conserved positives: loci with null alleles detected in the original data set which were not 802 confirmed within under-sampled data set.

803 


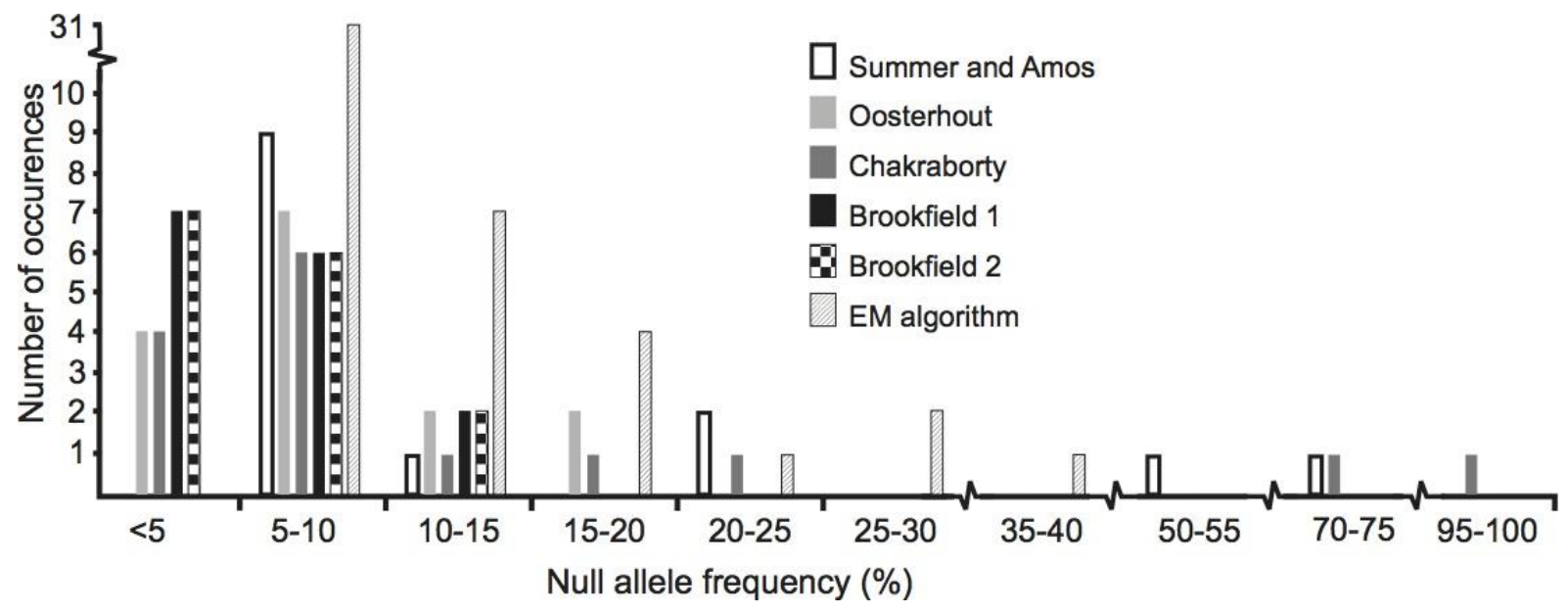

805 Figure 1

806 


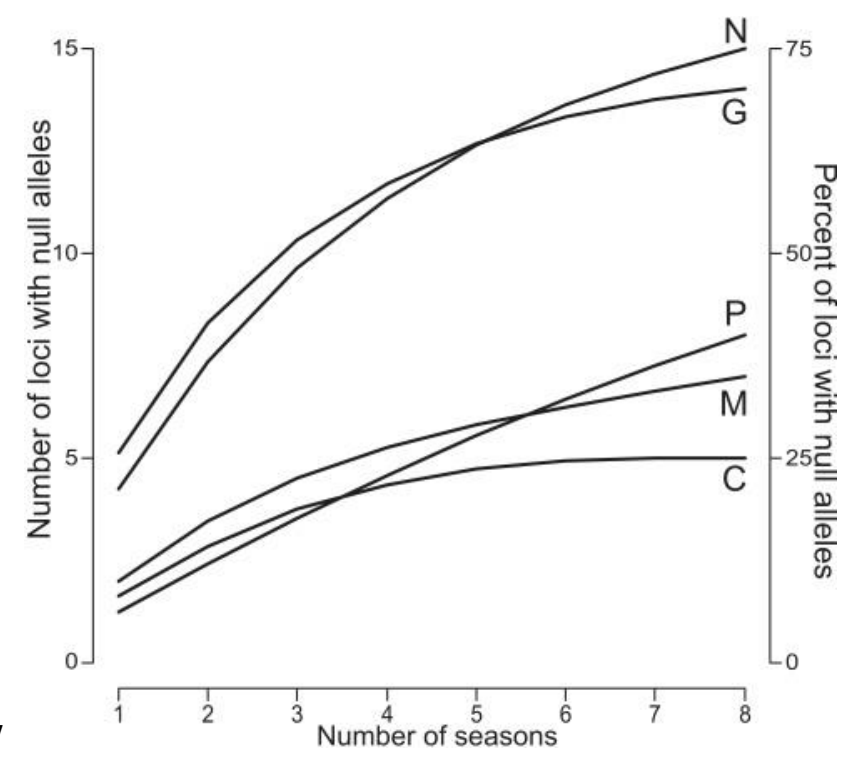

808 Figure 2

809 


\begin{tabular}{|c|c|c|c|c|}
\hline CERVUS & $22.4 \%$ & $57.9 \%$ & $31.6 \%$ & $33.3 \%$ \\
\hline $2.3 \%$ & GENEPOP & $24.0 \%$ & $28.1 \%$ & $12.0 \%$ \\
\hline $35.4 \%$ & $1.8 \%$ & MICRO- & $33.3 \%$ & $30.0 \%$ \\
\hline $13.6 \%$ & $2.9 \%$ & $25.3 \%$ & $\begin{array}{c}\text { MLCKER } \\
\text { NullFreq }\end{array}$ & $17.9 \%$ \\
& & & & \\
\hline- & - & - & - & $\begin{array}{c}\text { Parent- } \\
\text { offspring }\end{array}$ \\
\hline
\end{tabular}

811 Figure 3 


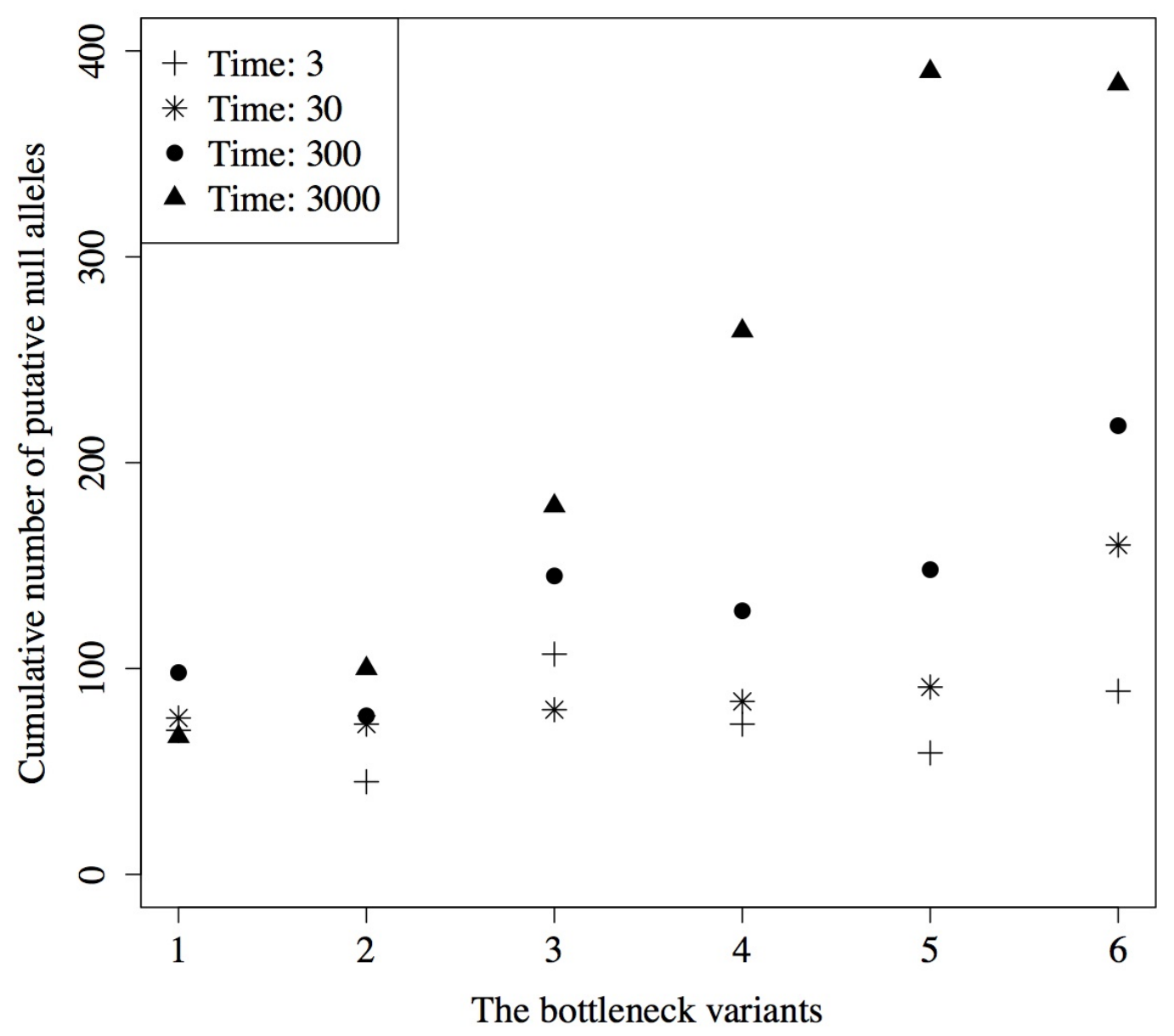

812

813 Figure 4 


\section{Supporting Information}

815 Additional Supporting Information may be found in the online version of this article:

816

817 Table S1 Characteristics of 20 microsatellite loci analysed in the root vole population, 818 organized in four multiplex PCR reactions.

819

820 Table S2 Loci with putative null alleles confirmed for the entire dataset (root vole population, 821 all years pooled) using all four methods.

822

823 Table S3 Characteristics of the simulated populations. Range, mean values and SDs were 824 computed separately for 50 replicates representing each scenario of the bottleneck size and 825 duration (in generations).

826

827 Table S5 Number of loci with null alleles detected using four programs in populations 828 simulated with various levels and duration of a bottleneck.

829

830 Fig. S1 The observed and expected frequency of homozygotes vs. null allele frequency in the 831 loci where null alleles were simulated using NullAlleleGenerator in 120 randomly selected 832 populations.

833

834 Table S4 Genetic estimates and null allele detection results computed for simulated 835 populations affected by bottleneck scenarios. 